Pacific Journal of Mathematic 


\title{
ON KILLING-RICCI FORMS OF LIE TRIPLE ALGEBRAS
}

\author{
MichIHIKo KIKKAWA
}

\begin{abstract}
The notion of Killing-Ricci forms of Lie triple algebras is introduced as a generalization of both of Killing forms of Lie algebras and the Ricci forms of the tangent Lie triple systems of Riemannian symmetric spaces. For a class of Lie triple algebras $\mathbb{B}$, it is shown that $\mathbb{B}$ is decomposed into a direct sum of simple ideals if its Killing-Ricci form is nondegenerate. As an application, structure of the reductive pair consisting of a semi-simple Lie algebra and its semi-simple subalgebra is investigated.
\end{abstract}

Introduction. The concept of Lie triple algebras has been introduced, originally, by $\mathrm{K}$. Yamaguti [11] as general Lie triple systems, related with locally reductive spaces of K. Nomizu [6], and treated by himself (e.g., [11]-[14]), A. A. Sagle (e.g., [8], [9]) and others. In the articles [2] and [3], the author considered Lie triple algebras as tangent algebras of homogeneous Lie loops or analytic homogeneous systems on manifolds. For the study of such algebraic systems on manifolds it seems to be very important to investigate the structure of real Lie triple algebras of finite dimension, as an extended analogy of the theory of Lie groups and Lie algebras. In this paper we consider the Killing-Ricci form $\beta$ of a Lie triple algebra (B), a symmetric bilinear form on (B) obtained by restricting the Killing form of the standard enveloping Lie algebra of $B$. Then, under an assumption by which $\beta$ becomes an invariant bilinear form on $(B)$, it is shown that a Lie triple algebra $\mathbb{B}$ is decomposed into a direct sum of simple Lie triple algebra ideals, if $\beta$ is nondegenerate (Theorem 2). This result is applied for a reductive pair of semisimple Lie algebra $\mathbb{Z}$ and semi-simple subalgebra $\Omega$ of $\mathbb{L}$, treated by A. A. Sagle [8], [9]. Then, a direct sum decomposition of $\mathbb{Z}$ into simple Lie triple algebras and semi-simple Lie algebra ideals of $\Re$ is obtained (Theorem 3).

1. Preliminaries. A Lie triple algebra (B) over a field $F$ is an anti-commutative algebra over $F$ whose multiplication is denoted by $X Y$ for $X, Y \in \mathbb{B S}$, with a trilinear operation $\mathbb{B S} \times$ BS $\times$ BS $\rightarrow$ \& denoted by $D(X, Y) Z$ satisfying the following conditions for $X, Y, Z, W \in \mathbb{B}$ :

(i) $D(X, X) Z=0$,

(ii) $\mathfrak{S}\{(X Y) Z+D(X, Y) Z\}=0$,

(iii) $\mathfrak{S} D(X Y, Z) W=0$, 
(iv) $D(X, Y)(Z W)=(D(X, Y) Z) W+Z(D(X, Y) W)$,

(v) $[D(X, Y), D(Z, W)]=D(D(X, Y) Z, W)+D(Z, D(X, Y) W)$, where $\subseteq$ denotes the cyclic sum with respect to $X, Y$ and $Z$. That is, Lie triple algebra is a synonym for a general Lie triple system introduced by K. Yamaguti [11]. Throughout this paper, we assume that (S) is a finite dimensional Lie triple algebra over a field of characteristic zero. The endomorphisms $D(X, Y)$ are called inner derivations of $\mathbb{S}$ and the Lie subalgebra $D(\mathbb{S},(\mathbb{S})$ of End(\$) generated by all inner derivations is called the inner derivation algebra of $\mathbb{B}$. The standard enveloping Lie algebra of $\mathbb{B}$ is a Lie algebra $\mathfrak{A}=\mathbb{B}+$ $D(B)$, (S) whose bracket operation is given as follows; $[X, Y]=X Y+$ $D(X, Y)$ for $X, Y \in \mathbb{B},[U, X]=-[X, U]=U X$ for $U \in D(\mathbb{B}, \mathbb{B}), X \in$ (B) and $[U, V]=U V-V U$ for $U, V \in D(\mathbb{B}$, (\&)). Thus $D(\mathbb{B}$, \&) is a Lie subalgebra of $\mathfrak{A}$ and $(\mathfrak{A}, D(\mathbb{S}, \mathbb{S}))$ forms a reductive pair. Conversely, for a Lie algebra $\mathcal{L}$ and a subalgebra $\Re$, if $(\mathfrak{R}, \mathfrak{R})$ is a reductive pair with the fixed decomposition $\mathscr{L}=\mathbb{B}+\Re,[\Omega, \mathbb{S}] \subset \mathbb{B}$, then CS $^{\prime}$ is a Lie triple algebra ([11]) under the operations $X Y=$ $[X, Y]_{\Theta}$ and $D(X, Y) Z=\left[[X, Y]_{\Omega}, Z\right]$ for $X, Y, Z \in \mathbb{B}$. The Lie triple algebra (B) is reduced to Lie algebra with $[X, Y]=X Y$ if $D(\mathbb{B},(\mathbb{S})=$ $\{0\}$, or it is reduced to Lie triple system with $[X, Y, Z]=D(X, Y) Z$ if $B S=\{0\}$. Conversely, every Lie algebra or Lie triple system is a Lie triple algebra as one of the reduced cases above. If $\mathbb{S B S}=\{0\}$ and $D(\mathbb{S}, \mathbb{B S})=\{0\}$, $\mathbb{S}$ is said to be abelian. A Lie triple subalgebra $\mathfrak{F}$ of $(\mathcal{S}$ is an invariant subalgebra if $D(\mathbb{S},(\mathbb{S}) \mathfrak{F} \subset \mathfrak{F}$, and an ideal if

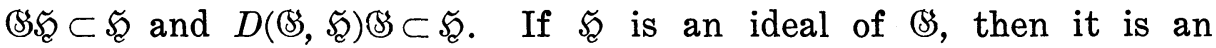
invariant subalgebra. Let $\mathscr{F}$ be an ideal of $\mathscr{B}$. A chain $\mathscr{S}=\mathscr{F}^{(0)} \supset$ $\mathfrak{S E}^{(1)} \supset \cdots \supset \mathfrak{S E}^{(i)} \supset \mathscr{S E}^{(i+1)} \supset \cdots$ of invariant subalgebras of $\mathbb{B}$ is defined

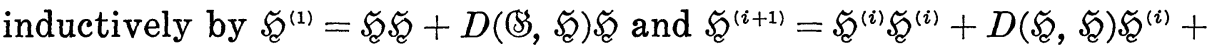
$D\left(\mathcal{S}, \mathscr{S}^{(i)}\right) \mathscr{S}^{(i)}$ for positive integers $i$ ([4]). Each $\mathfrak{S}^{(i+1)}$ is an ideal of $\mathfrak{S}^{(i)}$ and the quotient Lie triple algebra $\mathfrak{S E}^{(i)} / \mathscr{S}^{(i+1)}$ is abelian. An ideal $\mathfrak{S}$ of $\mathscr{S S}$ is said to be solvable if $\mathscr{S}^{(i)}=\{0\}$ for some integer $i$. The radical $\mathfrak{r}(\mathbb{S})$ of $\mathbb{B}$ is a (unique) maximal solvable ideal of $\mathbb{B}$. The Lie triple algebra (\$S is semi-simple if $\mathfrak{r}(\mathbb{S})=\{0\}$. The following facts have been shown in [4]:

(1.1) If $\mathbb{B S}$ is solvable then its standard enveloping Lie algebra $\mathfrak{A}=$ (S) $+D(\mathbb{B},(B)$ is a solvable Lie algebra and $D(\mathbb{B}$, (S) is a solvable Lie subalgebra of $\mathfrak{A}$.

(1.2) If $\mathfrak{A}=\mathbb{B}+D(\mathbb{S}, \mathbb{B})$ is a semi-simple Lie algebra then $\mathbb{B}$ is semi-simple.

A Lie triple algebra \& is simple if it has no nonzero proper ideal. It is easy to show the following: 
(1.3) 8 is simple if its standard enveloping Lie algebra is a simple Lie algebra.

2. Killing-Ricci forms, Let $\left\{E_{1}, E_{2}, \cdots, E_{n}\right\}$ be a basis of an $n$-dimensional Lie triple algebra $\&$, and $\left\{D_{1}, D_{2}, \cdots, D_{N}\right\}$ a basis of the inner derivation algebra $D(\mathbb{B}$, (B) if $D(\mathbb{B}, \mathbb{B}) \neq\{0\}$. For these bases we express the operations of $\mathbb{B S}$ as follows:

$$
\begin{aligned}
& E_{i} E_{j}=S_{i j}^{k} E_{k}, \quad D\left(E_{i}, E_{j}\right) E_{k}=R_{i j k}^{l} E_{l} \\
& D\left(E_{i}, E_{j}\right)=D_{i j}^{\alpha} D_{\alpha}, \quad\left[D_{\alpha}, E_{i}\right]=D_{\alpha} E_{i}=K_{\alpha i}^{j} E_{j},
\end{aligned}
$$

where the indices run through $1 \leqq i, j, k, l \leqq n$ and $1 \leqq \alpha \leqq N$. Denote by $\alpha$ the Killing form of the standard enveloping Lie algebra $\mathfrak{A}=\mathbb{B S}+D(\mathbb{S},(\mathbb{S})$. By the Killing-Ricci form $\beta$ of the Lie triple algebra $\mathbb{B}$ we mean a symmetric bilinear form on $\&$ determined by restricting $\alpha$ to $B S \times$ (S).

Proposition 1. For $X, Y \in \mathbb{B}$,

$$
\beta(X, Y)=\operatorname{tr} L(X) L(Y)+\operatorname{tr}(r(X, Y)+r(Y, X)),
$$

where $L(X)$ and $r(X, Y)$ are endomorphisms of \&S given by

$$
L(X) Y=X Y, \quad r(X, Y) Z=D(Z, X) Y \text { for } \quad X, Y, Z \in \mathbb{B} .
$$

Proof. If we set $S_{i j}=\operatorname{tr} L\left(E_{i}\right) L\left(E_{j}\right), R_{i j}=\operatorname{tr}\left(r\left(E_{i}, E_{j}\right)\right)$ and $\beta_{i j}=$ $\beta\left(E_{i}, E_{j}\right)$, it is sufficient to show the formula

$$
\beta_{i j}=S_{i j}+R_{i j}+R_{j i} \text { for } 1 \leqq i, j \leqq n .
$$

The expressions (2.1) with respect to the bases $\left\{E_{1}, \cdots, E_{n}\right\}$ and $\left\{D_{1}, \cdots, D_{N}\right\}$ imply the following:

$$
\begin{aligned}
& {\left[E_{i},\left[E_{j}, E_{k}\right]\right]=\left(S_{i m}^{l} S_{j k}^{m}-R_{j k i}^{l}\right) E_{l}+S_{j k}^{m} D_{i m}^{\alpha} D_{\alpha},} \\
& {\left[E_{i},\left[E_{j}, D_{\alpha}\right]\right]=-S_{i m}^{l} K_{\alpha j}^{m} E_{l}-D_{i m}^{\beta} K_{\alpha j}^{m} D_{\beta} .}
\end{aligned}
$$

Hence,

$$
\beta_{i j}=S_{i m}^{k} S_{j k}^{m}+R_{k j i}^{k}+D_{m i}^{\alpha} K_{\alpha j}^{m} .
$$

On the other hand, the expressions

$$
\begin{aligned}
& L\left(E_{i}\right) L\left(E_{j}\right) E_{k}=S_{i m}^{l} S_{j k}^{m} E_{l}, \\
& R_{k j i}^{l} E_{l}=D\left(E_{k}, E_{j}\right) E_{i}=D_{k j}^{\alpha} K_{\alpha i}^{l} E_{l}
\end{aligned}
$$

imply

$$
S_{i j}=S_{i m}^{k} S_{j k}^{m}, \quad R_{i j}=R_{k i j}^{k}=D_{k i}^{\alpha} K_{\alpha j}^{k}
$$


Therefore, (2.2) is obtained from (2.3) and (2.4).

REMARK 1. (1) If $B$ is reduced to Lie algebra, then $\beta$ is the Killing form of the Lie algebra $\&$. On the other hand, if $\mathbb{B}$ is reduced to Lie triple system, then $\beta(X, Y) / 2=(r(X, Y)+r(Y, X)) / 2$ is the Killing form of the Lie triple system (B) introduced by $\mathrm{T}$. Ravisankar [7].

(2) Suppose that (S) is a Malcev algebra. The Killing form $\theta$ of $\mathbb{B}$ introduced by 0 . Loos [5] is given by $\theta(X, Y)=\operatorname{tr}(\lambda(X) \lambda(Y))$, where $\lambda(X)$ denotes the left translation by $X$ in the Malcev algebra. K. Yamaguti [12] has shown that $(S)$ is a Lie triple algebra (general Lie triple system) under the operations $L(X)=\lambda(X), D(X, Y)=$ $\lambda(\lambda(X) Y)+[\lambda(X), \lambda(Y)]$. The Killing-Ricci form of this Lie triple algebra is equal to $5 \theta$. In [5], 0 . Loos considered the Malcev algebra (B) as a Lie triple system with the inner derivations $D(X, Y)=$ $2 \lambda(\lambda(X) Y)+[\lambda(X), \lambda(Y)]$. The Killing-Ricci form of this Lie triple system is equal to $6 \theta$ ([5, Lemma 6$]$ ).

REMARK 2. (1) Let $G$ be a Riemannian symmetric space. It is well known that the tangent space $\&$ at $e \in G$ is Lie triple system with respect to the ternary operation $[X, Y, Z]=R_{e}(X, Y) Z$, where $R$ denotes the curvature tensor of $G$. Then $R_{i j k}^{l}$ in (2.1) are the components of $R$ at $e$, and the Killing-Ricci form $\beta$ of the Lie triple system $\mathbb{B}$ has the components $\beta_{i j}=2 R_{i j}$, where $R_{i j}=R_{k i j}^{k}$ are the components of the Ricci tensor of $G$.

(2) Let $G$ be an analytic homogeneous Lie loop in [2] or, more generally, an analytic homogeneous system in [3], and $(B)$ be its tangent Lie triple algebra at $e \in G$. Then $S_{i j}^{k}$ and $R_{i j k}^{l}$ in (2.1) are respectively the components at $e$ of the torsion tensor and the curvature tensor of the canonical connection of $G$.

Now, let $\gamma$ be a trilinear form on $\mathbb{B S}$ given by

$$
\gamma(X, Y, Z)=\operatorname{tr}(D(X, Y) L(Z)) \text { for } X, Y, Z \in \mathbb{B} \text {. }
$$

It is evident that $\gamma$ vanishes identically if $\&$ is reduced to Lie algebra or reduced to Lie triple system.

Proposition 2. The Killing-Ricci form $\beta$ of a Lie triple algebra (8) satisfies the followings, for $X, Y, Z \in$ (S):

$$
\begin{gathered}
\beta(X Y, Z)+\beta(Y, X Z)=\gamma(Y, X, Z)+\gamma(Z, X, Y) \\
\beta(X, D(Y, Z) W)-\beta(D(W, X) Y, Z) \\
=\gamma(Y, Z, W X)-\gamma(W, X, Y Z)
\end{gathered}
$$




$$
\beta(X, D(Y, Z) W)+\beta(D(Y, Z) X, W)=0 .
$$

Proof. The Killing form $\alpha$ of $\mathfrak{A}=\mathbb{B}+D(\mathbb{S}$, (S) satisfies $\alpha([X, Y], Z)+\alpha(Y,[X, Z])=0$ for $X, Y, Z \in \mathbb{B}$. Since

$$
\alpha([X, Y], Z)=\beta(X Y, Z)+\gamma(X, Y, Z),
$$

the formula (2.6) is obtained. Applying (2.9) for

$$
\alpha(X,[[Y, Z], W])+\alpha([[X, W], Y], Z)=0
$$

we get

$$
\begin{aligned}
& \beta(X, D(Y, Z) W)+\beta(D(X, W) Y, Z) \\
&= \beta(X,(Y Z) W)+\beta((W X) Y, Z) \\
&+\gamma(Z Y, W, X)+\gamma(W X, Y, Z) .
\end{aligned}
$$

By using (2.6) for $\beta(X, W(Y Z))$ and $\beta(Y(X W), Z)$, we have

$$
\begin{aligned}
& \beta(X,(Y Z) W)+\beta((W X) Y, Z) \\
& =\gamma(X, W, Y Z)+\gamma(Y, Z, W X) \\
& \quad+\gamma(Y Z, W, X)+\gamma(X W, Y, Z) .
\end{aligned}
$$

The formula (2.7) is obtained from (2.10) and (2.11). Setting $X=W$ in (2.7), we get $\beta(X, D(Y, Z) X)=0$ which implies (2.8).

3. Semi-simple Lie triple algebras with $\gamma=0$. By an invariant form $b$ on a Lie triple algebra \&s we mean a symmetric bilinear form on $\mathbb{B}$ satisfying;

$$
\begin{gathered}
b(X Y, Z)+b(Y, X Z)=0 \\
b(X, D(Y, Z) W)-b(D(W, X) Y, Z)=0 \text { for } X, Y, Z, W \in \mathbb{S} .
\end{gathered}
$$

Proposition 3. Let $b$ be an invariant bilinear form on 8 . If $\mathfrak{K}$ is an invariant subalgebra of $G$, then $\mathfrak{S}^{\perp}=\{X \in \mathbb{S} ; b(X, \mathfrak{S})=0\}$ is an invariant subalgebra of $\mathbb{B}$. Moreover, if $\mathfrak{S}$ is an ideal of $\mathbb{S}$ then $\mathfrak{S}^{\perp}$ is an ideal.

Proof. From (3.2) the following is obtained:

$$
b(D(Y, Z) W, X)+b(W, D(Y, Z) X)=0 .
$$

Therefore, if $\mathfrak{S}$ is an invariant subalgebra of $\mathbb{S}$, then $D\left(\mathbb{S}, \mathfrak{S S}_{\mathfrak{S}} \mathfrak{K}^{\perp} \subset \mathfrak{S}^{\perp}\right.$. If $X, Z \in \mathbb{S S}$ and $Y \in \mathfrak{S E}^{\perp}$ then $b(X Y, \mathfrak{S})=b(Y, X \mathfrak{S})$ and $b(D(X, Y) Z, \mathfrak{S})=$ $b(D(Z, \mathfrak{S}) X, Y)$, which imply that $\mathfrak{S}^{\perp}$ is an ideal (resp. subalgebra) if $\mathfrak{S}$ is an ideal (resp. subalgebra) of the Lie triple algebra $\mathbb{S}$.

REMARK 3. If $\$ S$ is reduced to Lie triple system, then from 
(3.2) and (3.3) it follows that a symmetric bilinear form $b$ on $\$ 3$ is an invariant form if and only if $b$ is an invariant form of Lie triple system, in the sense of J. Wolf [10, (10.11)].

By a similar consideration of invariant bilinear forms on (8) as one for the cases of Lie triple system $[10, \S 10]$ and nonassociative algebras [1, p. 71], we have;

Proposition 4. Let $b$ be an invariant bilinear form on (\$S. Suppose that $b$ is nondegenerate. Then,

(1) The ideal $3^{\perp}$ for the center $3=\{X \in \mathbb{B S} X X \mathbb{S}=0$ and $D(X$, (B) $)=$

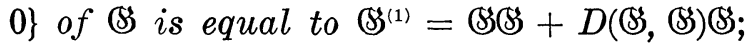

(2) If (S) has no nonzero ideal $\mathfrak{S E}$ satisfying $\mathfrak{S E S E}=\{0\}$ and $D(\mathbb{S}, \mathfrak{S}) \mathscr{K}=\{0\}$, then $\mathbb{B S}$ is decomposed into

$$
\text { (S) }=\mathscr{B S}_{1}+\mathscr{B S}_{2}+\cdots+\mathbb{B S}_{r}, \quad b=b_{1}+b_{2}+\cdots+b_{r},
$$

where $\mathbb{S}_{i}(1 \leqq i \leqq r)$ are simple ideals of $\mathbb{B}$ and $b_{i}=b \mid \mathbb{S}_{i} \times \mathbb{B}_{i}$, which $i s$ an invariant bilinear form on $\mathbb{S}_{i}$ for each $i$.

Proof. (1) is obtained from (3.1) and (2.2), and (2) is shown as follows. Let $\mathbb{B}_{1}$ be a minimal nonzero ideal in 8 . By Proposition 3, $\mathscr{S}_{1}^{\perp}$ is an ideal of $\mathbb{S}$ and so $\mathscr{S}=\mathbb{S}_{1} \cap \mathscr{S}_{1}^{\perp}$ is an ideal of $\mathbb{B S}$ contained in $\mathbb{S}_{1}$. If $X, Y \in \mathbb{B S}$ and $Z, W \in \mathfrak{F}$, then from (3.1) and (3.2) we have

$$
\begin{aligned}
& b(X, Z W)=b(X Z, W) \in b\left(\mathfrak{S}, \mathfrak{S}_{\mathcal{C}}\right)=\{0\}, \\
& b(X, D(Y, Z) W)=b(D(W, X) Y, Z) \in b\left(\mathfrak{S}, \mathfrak{F}_{\mathcal{C}}\right)=\{0\} .
\end{aligned}
$$

Since $b$ is supposed to be nondegenerate, $Z W=D(Y, Z) W=\{0\}$ for $Y \in \mathbb{S S}$ and $Z, W \in \mathfrak{S}$; i.e., $\mathfrak{S E S}_{\mathcal{E}}=\{0\}$ and $D(\mathbb{S}, \mathfrak{S}) \mathfrak{S}_{\mathcal{E}}=\{0\}$. Hence, by

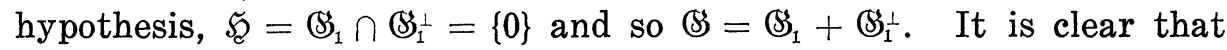
the bilinear form $b_{1}=b \mid \mathscr{S}_{1} \times \mathscr{S}_{1}$ is a nondegenerate invariant form on $\mathbb{B}_{1}$. The proposition is then established by induction on $\operatorname{dim}(\mathbb{S}$.

Now, let $\beta$ be the Killing-Ricci form of (s). If the trilinear form $\gamma$ given by (2.5) vanishes identically on $\mathbb{B}$, then from (2.6) and (2.7) it follows that $\beta$ is an invariant bilinear form on 8 .

THEOREM 1. Let (S) be a finite dimensional Lie triple algebra over a field of characteristic zero, on which the trilinear form $\gamma$ defined by (2.5) vanishes identically. Then;

(1) The Killing-Ricci form $\beta$ of \&s is nondegenerate if and only if the standard enveloping Lie algebra $\mathfrak{A}=\mathbb{B}+D(\mathbb{S},(\mathbb{S})$ is a semi-simple Lie algebra.

(2) If $\beta$ is nondegenerate, then (S) is a semi-simple Lie triple

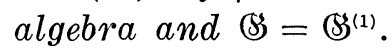


Proof. Since the Killing form $\alpha$ of the Lie algebra $\mathfrak{A}=\mathbb{B}+$ $D(\mathbb{S}$, (S) satisfies $\alpha(D(X, Y), Z)=\operatorname{tr} D(X, Y) L(Z)=\gamma(X, Y, Z)$ for $X, Y, Z \in \mathbb{B}$, the condition $\gamma=0$ implies that $\&$ and $D(\mathbb{B}$, (S) are orthogonal to each other with respect to $\alpha$. Hence,

$$
\begin{aligned}
\alpha(D(X, Y), D(Z, W)) & =\alpha([X, Y], D(Z, W)) \\
& =\beta(Y, D(Z, W) X) \text { for } X, Y, Z, W \in \mathbb{S} .
\end{aligned}
$$

Therefore, if $\beta$ is nondegenerate so is the restriction of $\alpha$ on $D(\mathbb{B},(\mathbb{S}) \times$ $D(\mathbb{S},(\mathbb{S})$, whence $\alpha$ itself is nondegenerate.

Conversely, if $\beta$ is degenerate, then, since $\beta$ is an invariant form on $\mathbb{B},(B) \perp=\{X \in \mathbb{B} ; \beta(X, \mathbb{B})=0\}$ is a nonzero ideal of $\mathbb{B}$ by Proposition 3 , and $B=\left(S^{\perp}+D\left(\mathbb{S}, \mathbb{S}^{\perp}\right)\right.$ is a Lie algebra ideal of $\mathfrak{A}$. By (3.4) we get

$$
\begin{aligned}
\alpha(\mathfrak{B}, \mathfrak{A}) & =\alpha\left(\mathbb{B}^{\perp}, \mathbb{S}\right)+\alpha\left(D\left(\mathbb{S}, \mathbb{B}^{\perp}\right), D(\mathbb{B}, \mathbb{S})\right) \\
& =\beta\left(\mathbb{S}^{\perp}, \mathbb{S}\right)+\beta\left(D\left(\mathbb{S}, \mathbb{S}^{\perp}\right)(\mathbb{S},(\mathbb{S})=\{0\} .\right.
\end{aligned}
$$

This shows that the Killing form $\alpha$ is degenerate, that is, $\mathfrak{A}$ is not semi-simple. Thus (1) is proved.

By (1.2), (B) is a semi-simple Lie algebra and so its center $3=$ $\{0\}$. Then (1) of Proposition 4 implies $\mathbb{S}=\mathbb{B S}^{(1)}$.

THEOREM 2. Let \&S be the same Lie triple algebra as in Theorem 1. Assume that the Killing-Ricci form $\beta$ of $\&$ is nondegenerate. Then $\&$ is decomposed into a direct sum of simple Lie triple algebra ideals $\mathbb{S}_{i}(1 \leqq i \leqq r)$ as follows;

$$
\mathbb{B}=\mathbb{B}_{1}+\mathbb{B}_{2}+\cdots+\mathbb{S}_{r} ; \beta=\beta_{1}+\beta_{2}+\cdots+\beta_{r},
$$

where $\beta_{i}$ is the Killing-Ricci form of $\mathbb{B}_{i}$ for each $i$. Moreover, the standard enveloping Lie algebra $\mathfrak{U}=\mathbb{B}+D(\mathbb{S}, \mathbb{B})$ is a direct sum of the standard enveloping Lie algebras $\mathfrak{A}_{i}=\mathbb{B}_{i}+D\left(\mathbb{S}_{i}, \mathbb{S}_{i}\right)$ of $\mathbb{S}_{i}$, each of which is a semi-simple Lie algebra ideal of $\mathfrak{A}$.

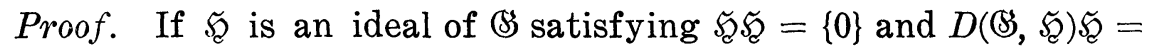

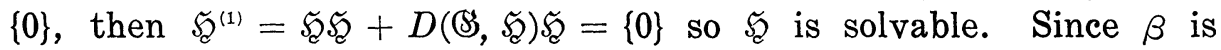
assumed to be nondegenerate, $\mathbb{S}$ is semi-simple by (2) in Theorem 1 , whence such an ideal $\mathfrak{S}$ must be $\{0\}$. Thus the assumptions of (2) in Proposition 4 are satisfied for the invariant bilinear form $\beta$. Therefore, $\mathbb{B}$ is a direct sum of simple ideals $\mathbb{B}_{i}(1 \leqq i \leqq r)$ and $\beta=$ $\beta_{1}+\cdots+\beta_{r}, \beta_{i}=\beta \mid \mathbb{S}_{i} \times \mathbb{S}_{i}$. If $X_{i} \in \mathbb{B}_{i}, Y_{j} \in \mathbb{S}_{j}$ and $Z_{k} \in \mathbb{S}_{k}$, then $D\left(X_{i}, Y_{j}\right) Z_{k} \in \mathbb{S}_{i} \cap \mathbb{S}_{j} \cap \mathbb{S}_{k}$. Hence $D\left(X_{i}, Y_{j}\right)=0$ and $D\left(X_{i}, Y_{i}\right) \mid \mathbb{S}_{j}=0$ for $i \neq j$. Thus we get $D(\mathbb{S}, \mathbb{S})=D\left(\mathbb{S}_{1}, \mathbb{S}_{1}\right)+\cdots+D\left(\mathbb{S}_{r}, \mathbb{S}_{r}\right), \mathfrak{A}=$ $\mathbb{B}+D(\mathbb{S}, \mathbb{S})=\mathfrak{A}_{1}+\cdots+\mathfrak{A}_{r}$, where $\mathfrak{A}_{i}=\mathbb{S}_{i}+D\left(\mathbb{S}_{i}, \mathbb{S}_{i}\right)$ are ideals 
of $\mathfrak{A}$, and $\beta_{i}=\beta\left|\mathscr{S}_{i} \times \mathscr{S}_{i}=\alpha\right| \mathscr{B}_{i} \times \mathscr{S}_{i}=\alpha \mid \mathscr{S}_{i} \times \mathscr{S}_{i}$, where $\alpha_{i}$ denotes the Killing form of the Lie algebra $\mathfrak{A}_{i}$ for each $i$.

4. Applications for pairs of semi-simple Lie algebras. Let $\Re$ be a semi-simple Lie subalgebra of a semi-simple Lie algebra $\&$ over a field of characteristic zero, where $\mathfrak{L}$ is assumed to be of finite dimension. A. Sagle $[8,9]$ has shown that the pair $(\Omega, R)$ is then a reductive pair with a decomposition $\mathbb{L}=\mathbb{B}+\Re$, where $\mathbb{S}=\{X \in \mathbb{R}$; $\operatorname{Kill}_{\mathfrak{R}}(X, \Re)=0$ \} and $\mathrm{Kill}_{\mathfrak{R}}$ denotes the Killing form of $\mathfrak{R}$, and the bilinear form $\beta$ on $\mathbb{S}$ given by $\beta(X, Y)=\operatorname{Kill}_{\Omega}(X, Y)$ for $X, Y \in \mathbb{S}$ is nondegenerate. For brevity of discussion assume that $\Omega$ contains no nonzero ideal of $\mathbb{R}$. Then $\Re$ may be identified with a Lie subalgebra of the Lie algebra End(\$S). For $X, Y \in \mathbb{S}$ let $[X, Y]=X Y+D(X, Y)$, where $X Y=[X, Y]_{\&}$ and $D(X, Y)=[X, Y]_{\AA}$ are the projections of $[X, Y]$ into $\mathbb{S}$ and $\Re$, respectively. Since $(\Omega, \Omega)$ is a reductive pair, (83 is a Lie triple algebra with the operations $X Y$ and $D(X, Y) Z=$ $[D(X, Y), Z]$ for $X, Y, Z \in \mathbb{B}$ ([13]). The standard enveloping Lie algebra $\mathfrak{A}=\mathbb{B}+D(\mathbb{S}, \mathbb{S})$ of this Lie triple algebra $\mathbb{S}$ is an ideal of $\&$ and the inner derivation algebra $D(\mathbb{S}, \mathbb{S})$ is an ideal of $\Re$. Hence the Killing form $\alpha$ of $\mathscr{A}$ is the restriction of the Killing form of $\mathbb{Q}$ to $\mathfrak{A} \times \mathfrak{A}$, and so $\gamma(X, Y, Z)=\alpha(D(X, Y), Z) \in \operatorname{Kill}_{\varepsilon}(\mathbb{S}, \Re)=\{0\}$ for $X, Y$, $Z \in \mathbb{B}$. The nondegenerate bilinear form $\beta$ is equal to the KillingRicci form of the Lie triple algebra $\$$. Therefore, from Theorem 2 it follows that $B$ is decomposed into a direct sum of simple ideals $\mathbb{S}_{i}(1 \leqq i \leqq r)$ of $\mathbb{S}$. Each $\mathscr{S}_{i}$ is ad $\Re$-invariant since $\beta\left(\mathscr{S}_{i},\left[\Re, \mathscr{S}_{j}\right]\right)=0$ for $i \neq j$. In fact, by using Jacobi's identity in $\AA$ and the fact $D\left(\mathbb{S}_{i}, \mathbb{S}_{j}\right)=\{0\}$ for $i \neq j$, we can show ad $\mathbb{S}_{i}$ ad $\left[\Re, G_{j}\right](\mathbb{S}+D(\mathbb{S},(\mathbb{S})) \subset$ $\mathbb{S}_{i} \cap \mathbb{S}_{j}+D\left(\mathbb{S}, \mathbb{S}_{i}\right) \cap D\left(\mathbb{S}, \mathbb{S}_{j}\right)=\{0\}$ for $i \neq j$. If $\mathbb{S}_{i}$ is reduced to Lie algebra for some $i$, then $D\left(\mathbb{S}_{i}, \mathbb{S}\right)=\{0\}$ and so $\mathbb{S}_{i}$ is a Lie algebra ideal of $\mathbb{L}$. Let $\Omega_{0}$ be an ideal of $\Omega$ such that $\Omega=D(\mathbb{S}, \mathbb{S})+\Re_{0}$. In case $\Omega$ contains nonzero ideals of $\mathcal{L}, \mathbb{L}$ and $\Omega$ should be factored by the maximal ideal $\Omega_{0}$ among them.

Summing up the arguments above, we obtain from Theorem 2 the following;

TheORem 3. Let $\Omega$ be a semi-simple Lie subalgebra of a finite dimensional semi-simple Lie algebra $\mathfrak{Q}$ over a field of characteristic zero. Then $\&$ is decomposed as follows:

$$
\mathfrak{L}=\mathbb{S}_{1}+\cdots+\mathbb{B}_{r}+\mathfrak{D}_{1}+\cdots+\mathfrak{D}_{r}+\mathfrak{\Omega}_{0}+\mathfrak{L}_{0},
$$

where $\mathscr{S}_{i}(1 \leqq i \leqq r)$ are simple Lie triple algebras which are ad $\Re$ invariant in $\mathbb{R}, \mathfrak{D}_{i}=D\left(\mathbb{S}_{i}, \mathbb{S}_{i}\right)$ are their inner derivation algebras some of which might be zero that are reduced to Lie algebra ideals 
of $\&, \mathbb{R}_{0}$ is the maximal ideal of $\mathbb{2}$ contained in $\Re$, and $\Re_{0}$ is the complmeentary ideal of the ideal $\mathfrak{D}_{1}+\cdots+\mathfrak{D}_{r}+\mathfrak{S}_{0}$ in $\Re$, each of $\mathfrak{D}_{i}$ being an ideal of $\Omega$.

For each $i$, the standard enveloping Lie algebra $\mathfrak{A}_{i}=\mathscr{S}_{i}+\mathfrak{D}_{i}$ is a semi-simple ideal of the Lie algebra $\Omega$.

\section{REFERENCES}

1. N. Jacobson, Lie Algebras, Interscience, 1962.

2. M. Kikkawa, Geometry of homogeneous Lie loops, Hiroshima Math. J., 5 (1975), 141-179.

3. - On homogeneous systems I, II, Mem. Fac. Lit. Sci. Shimane Univ., Nat. Sci., 11 (1977), 9-12; Mem. Fac. Sci. Shimane Univ., 12 (1978), 5-13.

4. - Remarks on solvability of Lie triple algebras, Mem. Fac. Sci. Shimane Univ., 13 (1979), 17-22.

5. O. Loos, Über eine Beziehung zwischen Malcev-Algebren und Lie Tripelsystemen, Pacific J. Math., 18 (1966), 553-562.

6. K. Nomizu, Invariant affine connections on homogeneous spaces, Amer. J. Math., 76 (1954), 33-65.

7. T. Ravisankar, Some remarks on Lie triple system, Kumamoto J. Sci., (Math.), 11 (1974), 1-8.

8. A. Sagle, Jordan algebras and connections on homogeneous spaces, Trans. Amer. Math. Soc., 187 (1974), 1-8.

9. A. Sagle and D. Winter, On homogeneous spaces and reductive subalgebras of simple Lie algebras, Trans. Amer. Math. Soc., 128 (1967), 142-147.

10. J. Wolf, On the geometry and classification of absolute parallelisms, II, J. Differential Geometry, 7 (1972/1973), 19-44.

11. K. Yamaguti, On the Lie triple system and its generalization, J. Sci. Hiroshima Univ., A 21 (1958), 155-160.

12. - Note on Malcev algebras, Kumamoto J. Sci., A 5 (1962), 203-207.

13. - On the theory of Malcev algebras, Kumamoto J. Sci., A 6 (1963), 9-45.

14. - On cohomology groups of general Lie triple systems, Kumamoto J. Sci., A 8 (1969), 135-146.

Received May 30, 1980.

SHIMANE UNIVERSITY

Matsue, Shimane

JAPAN 



\section{PACIFIC JOURNAL OF MATHEMATICS}

\section{EDITORS}

DONALD BABBITT (Managing Editor)

University of California

Los Angeles, California 90024

Hugo RossI

University of Utah

Salt Lake City, UT 84112

C. C. MOORE and ANDREW OGG

University of California

Berkeley, CA 94720

\section{J. DugundJI}

Department of Mathematics University of Southern California Los Angeles, California 90007

R. FinN and J. Milgram Stanford University Stanford, California 94305

\section{ASSOCIATE EDITORS}
R. ARENS
E. F. BECKENBACH
B. H. Neumann
F. WOLF
K. YoshidA

\section{SUPPORTING INSTITUTIONS}

UNIVERSITY OF ARIZONA

UNIVERSITY OF BRITISH COLUMBIA

CALIFORNIA INSTITUTE OF TECHNOLOGY

UNIVERSITY OF CALIFORNIA

MONTANA STATE UNIVERSITY

UNIVERSITY OF NEVADA, RENO

NEW MEXICO STATE UNIVERSITY

OREGON STATE UNIVERSITY
UNIVERSITY OF OREGON

UNIVERSITY OF SOUTHERN CALIFORNIA

STANFORD UNIVERSITY

UNIVERSITY OF HAWAII

UNIVERSITY OF TOKYO

UNIVERSITY OF UTAH

WASHINGTON STATE UNIVERSITY

UNIVERSITY OF WASHINGTON 


\section{Pacific Journal of Mathematics}

\section{Vol. 96, No. $1 \quad$ November, 1981}

Hédi Amara, Groupe des classes et unité fondamentale des extensions quadratiques relatives à un corps quadratique imaginaire principal $\ldots \ldots \ldots 1$

Douglas S. Bridges, On the isolation of zeroes of an analytic function $\ldots \ldots 13$ Andrew J. Casson and John L. Harer, Some homology lens spaces which

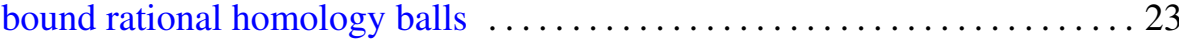

Z. A. Chanturia, On the absolute convergence of Fourier series of the classes $H^{\omega} \cap V[v]$

J.-F. Colombeau and Mário Carvalho Matos, On some spaces of entire functions defined on infinite-dimensional spaces $\ldots \ldots \ldots \ldots \ldots \ldots \ldots 63$

Edwin Duda, Pointwise periodic homeomorphisms on chainable continua . . .77

Richard F. Gustafson, A simple genus one knot with incompressible spanning surfaces of arbitrarily high genus $\ldots \ldots \ldots \ldots \ldots \ldots \ldots \ldots 1$

Fumio Hiai, Masanori Ohya and Makoto Tsukada, Sufficiency, KMS condition and relative entropy in von Neumann algebras

Ted Hurley, Intersections of terms of polycentral series of free groups and free Lie algebras. II .................................. 111

Robert Edward Jamison, II, Partition numbers for trees and ordered sets . . 115 R. D. Ketkar and N. Vanaja, A note on FR-perfect modules ............. 141 Michihiko Kikkawa, On Killing-Ricci forms of Lie triple algebras ....... 153

Jorge Lewowicz, Invariant manifolds for regular points 163

Richard W. Marsh, William H. Mills, Robert L. Ward, Howard Rumsey and Lloyd Richard Welch, Round trinomials .....

Claude Schochet, Topological methods for $C^{*}$-algebras. I. Spectral sequences

Yong Sian So, Polynomial near-fields?

Douglas Wayne Townsend, Imaginary values of meromorphic functions in the disk

Kiyoshi Watanabe, Coverings of a projective algebraic manifold .. 243

Martin Michael Zuckerman, Choosing $l$-element subsets of $n$-element sets 